\title{
Abnormal levels of antioxidant defenses in a large sample of patients with hypertensive disorders of pregnancy
}

Maria Luisa Miranda Guisado, Antonio J Vallejo-Vaz, Pablo Stiefel García Junco, Luis Jiménez Jiménez, Salvador García Morillo, Ovidio Muñiz Grijalvo, Verónica Alfaro Lara, José Villar Ortiz and Encarnación Pamies-Andréu

Increased levels of oxidative stress have been demonstrated in Preeclampsia in previous studies, but this finding has not been established in other hypertensive disorders in pregnancy (HDP). We measured different markers of lipid peroxidation and antioxidant defenses by spectrophotometry or enzymoimmunoanalysis in 339 pregnant women: 85 with gestational hypertension (GH), 88 chronic hypertension (CH), 104 Preeclampsia and 62 healthy pregnant control women (PCW). Lower activity of superoxide dismutase and higher levels of catalase were found in $\mathrm{GH}, \mathrm{CH}$ and preeclampsia compared with PCW (964.4 $\pm 116.5,970.0 \pm 120.4,971.2 \pm 137.5$ and $1063.4 \pm 133.7 \mathrm{U} \mathrm{g}^{-1} \mathrm{Hb}, P<0.001$; and $313.0 \pm 71.7,292.2 \pm 45.3$, $297.1 \pm 47.2,215.5 \pm 26.2 \mathrm{U} \mathrm{mg}^{-1} \mathrm{Hb}, P<0.0001$; respectively). Regarding the glutathione REDOX cycle, we found the following in GH, $\mathrm{CH}$ and preeclampsia compared with PCW: a decrease in its reduced form $(2.6 \pm 0.6,2.7 \pm 0.8,2.7 \pm 0.9$, $\left.3.3 \pm 1.3 \mu \mathrm{mol} \mathrm{I}^{-1}, P<0.003\right)$, a parallel increase in the oxidized form $(185.6 \pm 68.9,194.7 \pm 75.0,184.3 \pm 78.3$, $\left.85.1 \pm 27.5 \mu \mathrm{mol} \mathrm{I}^{-1}, P<0.0001\right)$ and an increment in glutathione peroxidase $(85.9 \pm 22.0,86.4 \pm 20.9,82.1 \pm 23.5$ and $\left.77.2 \pm 19.7 \mathrm{U} \mathrm{g}^{-1} \mathrm{Hb}, P<0.04\right)$ and glutathione reductase $(6384.3 \pm 1261.9,6724.6 \pm 1154.1,6287.9 \pm 1399.9$ and $6044.4 \pm 1208.4 \mathrm{mU} \mathrm{g}^{-1} \mathrm{Hb}, P<0.01$, respectively). Nitrites/nitrates were higher in patients with preeclampsia than in PCW $\left(31.50 \pm 15.08,26.80 \pm 8.39 \mu \mathrm{mol} \mathrm{I}^{-1}, P<0.002\right)$. Although malondialdehyde and oxidized-LDL levels were similar among groups, free fatty acids were increased in every HDP (GH 514.6 \pm 194.6 , CH $501.3 \pm 197.4$, preeclampsia $555.2 \pm 230.1 \mu \mathrm{mol} \mathrm{I}^{-1}$ ) compared with PCW (351.4 $\left.\pm 146.1 \mu \mathrm{mol} \mathrm{I}^{-1}\right), P<0.0001$. Our results show an oxidation/reduction imbalance with an increase in oxidative stress coupled with a decreased capacity of antioxidant systems, not only in preeclampsia but also in every HDP.

Hypertension Research (2012) 35, 274-278; doi:10.1038/hr.2011.200; published online 24 November 2011

Keywords: antioxidant status; hypertensive disorders of pregnancy; oxidative stress; preeclampsia

\section{INTRODUCTION}

Hypertensive disorders of pregnancy (HDP) can be classified into different types: chronic hypertension $(\mathrm{CH})$, which is defined as hypertension present before pregnancy or diagnosed before the 20th week of gestation, as well as cases of hypertension diagnosed for the first time during pregnancy that do not resolve postpartum and persist $>12$ weeks after delivery; preeclampsia, which is defined as the development of hypertension after 20 weeks of gestation, accompanied by proteinuria of $\geqslant 300 \mathrm{mg}$ in $24 \mathrm{~h}$, that is resolved after gestation; and transient gestational hypertension $(\mathrm{GH})$, which displays a similar time of onset as preeclampsia, but is not marked by proteinuria and is resolved within 3 months of delivery. ${ }^{1}$ Preeclampsia is the most specific and best-studied HDP and it is associated with the highest maternal and fetal morbidity and mortality. ${ }^{1}$ The etiology of preeclampsia has been largely studied but remains yet unknown, probably because it depends on a multifactorial basis with a common final pathway, which leads to a multisystemic syndrome characterized by vasoconstriction, metabolic changes, endothelial dysfunction, activation of the coagulation cascade, increased inflammatory response and reduced organ perfusion. ${ }^{2}$

Key mechanisms for maternal endothelial dysfunction in preeclampsia are not well understood, but poor placentation, disturbed trophoblast invasion and reduced placental perfusion are thought to be important predisposing factors. ${ }^{3,4}$ Oxidative stress, characterized by excessive production of reactive oxygen species, and an inadequate or overwhelmed antioxidant defense mechanisms have been proposed as

Unidad Clínico-Experimental de Riesgo Vascular (UCERV- UCAMI), Department of Internal Medicine, Virgen del Rocío University Hospital, and Instituto de Biomedicina (IBiS), Sevilla, Spain

Correspondence: Dr E Pamies-Andréu, Unidad Clínico-Experimental de Riesgo Vascular (UCERV- UCAMI), Department of Internal Medicine, Virgen del Rocío University Hospital, and Instituto de Biomedicina (IBiS), Avda Manuel Siurot s/n 41013, Sevilla, Spain.

E-mail: encarnacion.pamies.sspa@juntadeandalucia.es

Received 13 July 2011; revised 9 August 2011; accepted 14 August 2011; published online 24 November 2011 
important mechanisms. ${ }^{5,6}$ In this way, increased oxidative stress have been found to be elevated in women with preeclampsia and even persists postpartum when compared with normotensive pregnant women. ${ }^{4,7}$ It has been suggested that products as trophoblastic fragments, superoxide anion and other free radicals, cytokines, eicosanoids or others can be released by the ischemic placenta. ${ }^{3}$ These products act on a vulnerable to oxidative stress maternal endothelium, stimulating cytokine production, adhesion molecules, tumor necrosis factors and neutrophils. ${ }^{8}$ These agents will then act as mediators for a systemic inflammatory response and for the endothelial dysfunction that could lead to the characteristic diffuse vasospasm of preeclampsia.

In the past few years, several authors have documented some changes in the capacity of antioxidant systems in preeclampsia as consequence of the increase in oxidative stress, ${ }^{9}$ but this subject has not been studied in other forms of HDP.

The aim of our study was to assess the suspected imbalance between oxidative stress and the ability of antioxidant defense systems in a large sample of pregnant women with HDP compared with healthy pregnant control women (PCW), and to establish whether these findings are only present in preeclampsia or can also be found in every HDP.

\section{METHODS}

\section{Study population}

We included 339 consecutive caucasian pregnant women between 18 and 40 years old, attended in a Women's Hospital (Hospitales Universitarios Virgen del Rocio, Sevilla, Spain). Distribution was as follows: 85 cases of GH, $88 \mathrm{CH}, 104$ preeclampsia and 62 PCW. Hypertension was established by measuring blood pressure twice 1 week apart (three times each in a seated position after $5 \mathrm{~min}$ in a relaxed condition). The diagnosis was classified according to the guidelines of the National High Blood Pressure Education Program Working Group on High Blood Pressure in Pregnancy. ${ }^{1}$

\section{Exclusion criteria}

Women with lipid disorders, diabetes or smoking habit were excluded. We also excluded those women under treatment with agents that modify endothelial function as well as those suffering any malignancy, chronic consumptive disease, chronic kidney disease or immunological diseases. The only antihypertensive treatment allowed at the time to obtain the samples was methyldopa.

The procedures followed in this study were in accordance to institutional guidelines, all subjects gave informed consent and our Local Ethics Committee approved the study protocol.

\section{Design}

This was an observational, cross sectional and comparative study carried out in a large sample of women with hypertensive disorders of pregnancy and a control group of healthy pregnant women.

\section{Anthropometric variables}

Age, height and weight were measured and body mass index was then calculated. Fetal weight at birth and blood pressure were also recorded.

\section{Laboratory parameters}

Blood samples were obtained after an overnight fast of $12 \mathrm{~h}$ and were performed between the 28th and 37th week of gestation. In every subject the following laboratory parameters were analyzed: oxidized-low-density lipoprotein (ox-LDL), free fatty acids (FFA), Nitrites/nitrates and total antioxidant status (TAS) in plasma; superoxide dismutase (SOD), glutation peroxidase (GPX) and glutation reductase (GRX) in erythrocytes. In addition, in 48 cases of $\mathrm{GH}, 56 \mathrm{CH}, 43$ preeclampsia and $38 \mathrm{PCW}$ we measured malondialdehyde, oxidized gluthation (GSSG) and reduced gluthation (GSH) in plasma and glucose-6-phosphate dehydrogenase (G6PDH) and catalase in erythrocytes.
Ox-LDL was determined by LAB ELISA technique (quantitative measurement of serum human antibodies against ox-308171392LDL (Biomedica group, Vienna, Austria)..$^{10}$ FFA were determined using an enzymo-spectrophotometric method, based in the quantification of the amount of AMP generated as the result of the formation of acetyl-CoA in the presence of acetyl-CoA-synthase and ATP. ${ }^{11}$ For measurement of nitrites/nitrates level, a colorimetric method based on Greiss' reaction was used. ${ }^{12}$ TAS capacity was determined in blood venous samples centrifugated for $5 \mathrm{~min}$ at 3000 r.p.m. to separate plasma, using a TAS Kit (Randox Laboratories Ltd, Antrim, UK). ${ }^{13}$ GPX, GRX and intracellular SOD were determined in three times washed and lysed erythrocytes using the respective kits provided by Ramdox laboratories for these enzymes. Erythrocytes' fragments after hemolysis were cultured in the presence of a known concentration of hydrogen peroxide $\left(\mathrm{H}_{2} \mathrm{O}_{2}\right){ }^{14-16}$

GSSG and GSH were determined by the GSH/GSSG-412 assay that included the thiol-scavenging reagent, 1-methyl-2-vinylpyridinium trifluromethasulfonate at a level that rapidly scavenges GSH and prevent the oxidation of GSH to GSSG during sample preparation. The method uses Ellman's reagent $\left(5,5^{\prime}\right.$ dithiobis-2-nitrobenzoic acid), which reacts with GSH to form a spectrophotometrically detectable product at $412 \mathrm{~nm} \cdot{ }^{17}$ Catalase activity was measured at $20^{\circ} \mathrm{C}$ by following the degradation of the substrate $\mathrm{H}_{2} \mathrm{O}_{2}$ spectrophotometrically monitored at $240 \mathrm{~nm}$. Using a molar extinction coefficient, the rate at the first $30 \mathrm{~s}$ was used to calculate the activity in units. ${ }^{18}$ Malondialdehyde: this oxidazing lipid degradation final product, under low $\mathrm{pH}$ and high-temperature conditions, participates in a nucleophilic reaction with thiobarbituric acid generating an orange colored compound that may be spectrophotometrically assessed at $532 \mathrm{~nm} .{ }^{19} \mathrm{G} 6 \mathrm{PDH}$ was determined by using an enzymatic spectrophotometric method. ${ }^{20}$

\section{Statistical analysis}

A $P$-value $<0.05$ was considered as statistically significant. To compare means, analysis of variance was used for normally distributed variables and the Mann-Whitney test for non-normally distributed variables. Previously, normality of distribution was studied using the Kolmogorow-Smirnoff test. The SPSS statistical software (SPSS, IBM Company) was used. Data are expressed as means \pm s.d. throughout the text.

\section{RESULTS}

The studied sample included 277 women with HDP (GH: 85, CH: 88, preeclampsia: 104) and 62 PCW. General characteristics for the studied sample are shown in Table 1 . Women with $\mathrm{CH}$ were older and had a higher body mass index than the other three groups, but blood pressure levels were lower and fetal weight at the end of the pregnancy was higher in $\mathrm{CH}$ than in patients with preeclampsia.

Parameters of oxidation and antioxidation are expressed in Tables 2 and 3. Levels of malondialdehyde, catalase, GSSG, GSH and G6PDH were available only in 185 women $(\mathrm{GH} \mathrm{48;} \mathrm{CH}, 56$; preeclampsia 43; PCW 38). In short, we observed a decreased activity of SOD and an increased activity of catalase in all HDP as compared with PCW. TAS levels were lower in $\mathrm{CH}$ than in the other groups. Regarding the glutathione REDOX cycle, GSH levels were lower and in parallel GSSG levels were higher in all HDP compared with PCW. GPX and GRX

Table 1 Anthropometric characteristics

\begin{tabular}{lccccc}
\hline & GH & CH & $P C L$ & $C P W$ & P-value \\
\hline Age (years) & $30.3 \pm 5.2$ & $31.7 \pm 4.0$ & $29.5 \pm 6.3$ & $27 \pm 7.8$ & $P=0.04$ \\
BMI (kg m -2$)$ & $31.1 \pm 4.3$ & $32.4 \pm 6.8$ & $28.4 \pm 5.1$ & $25 \pm 5.3$ & $P<0.001$ \\
SBP (mm Hg) & $151.2 \pm 10.1$ & $154.7 \pm 18.2$ & $160 \pm 16.5$ & $110 \pm 16$ & $P<0.001$ \\
DBP (mm Hg) & $96.4 \pm 7.4$ & $98 \pm 9.3$ & $101.7 \pm 8.7$ & $66.6 \pm 11.5$ & $P<0.001$ \\
FW (g) & $2928 \pm 530$ & $3468.7 \pm 354$ & $2165.4 \pm 857.6$ & $3011 \pm 615$ & $P=0.001$
\end{tabular}

Abbreviations: BMI, body mass index; $\mathrm{CH}$, chronic hypertension; $\mathrm{CPW}$, control pregnant women; DBP, diastolic blood pressure; FW, fetal weight; GH, gestational hypertension; SBP, systolic blood pressure; PCL, preeclampsia. 
Table 2 Oxidative markers

\begin{tabular}{|c|c|c|c|c|c|}
\hline & $G H$ & $\mathrm{CH}$ & $P C L$ & $C P W$ & P-value \\
\hline FFA $(\mu \mathrm{mol} \mathrm{I-1})$ & $514.6 \pm 194.6$ & $501.3 \pm 197.4$ & $555.2 \pm 230.1$ & $351.4 \pm 146.1$ & $P<0.000$ \\
\hline $\mathrm{MDA}^{\mathrm{a}}\left(\mathrm{nmol} \mathrm{I}^{-1}\right)$ & $1.5 \pm 0.3$ & $1.6 \pm 0.3$ & $1.7 \pm 0.5$ & $1.6 \pm 0.4$ & NS \\
\hline
\end{tabular}

Abbreviations: $\mathrm{CH}$, chronic hypertension; CPW, control pregnant women; FFA, free fatty acids; GH, gestational hypertension; MDA, malondialdehyde; NS, not significant; Ox-LDL, oxidized-LDL; $\mathrm{PCL}$, preeclampsia.

${ }^{\mathrm{a}} \mathrm{N}=185$.

Table 3 Antioxidant markers

\begin{tabular}{|c|c|c|c|c|c|}
\hline & $G H$ & $\mathrm{CH}$ & $P C L$ & $C P W$ & $\mathrm{P}$-value \\
\hline TAS $\left(\mu \mathrm{mol} \mathrm{I}^{-1}\right)$ & $1108.2 \pm 105.2$ & $1092.4 \pm 88.2$ & $1141.4 \pm 123.5$ & $1126 \pm 72.9$ & $P=0.008$ \\
\hline GPX $\left(\mathrm{Ug}^{-1} \mathrm{Hb}\right)$ & $85.9 \pm 22$ & $86.4 \pm 20.9$ & $82.1 \pm 23.5$ & $77.2 \pm 19.7$ & $P=0.04$ \\
\hline GRX $\left(m U g^{-1} \mathrm{Hb}\right)$ & $6384.3 \pm 261.9$ & $6724.6 \pm 1154.1$ & $6287.9 \pm 1399.9$ & $6044.4 \pm 1208.4$ & $P=0.01$ \\
\hline $\mathrm{SOD}\left(\mathrm{Ug}^{-1} \mathrm{Hb}\right)$ & $964.4 \pm 116.5$ & $970 \pm 120.4$ & $971.2 \pm 137.5$ & $1063.4 \pm 133.7$ & $P<0.01$ \\
\hline $\mathrm{CAT}^{\mathrm{a}}\left(\mathrm{U} \mathrm{mg}^{-1} \mathrm{Hb}\right)$ & $313 \pm 71.7$ & $292.2 \pm 45.3$ & $297.1 \pm 47.2$ & $215.5 \pm 26.2$ & $P<0.001$ \\
\hline $\mathrm{GSSG}^{\mathrm{a}}\left(\mu \mathrm{mol} \mathrm{I}^{-1}\right)$ & $185.6 \pm 68.9$ & $194.7 \pm 75$ & $184.3 \pm 78.3$ & $85.1 \pm 27.5$ & $P<0.001$ \\
\hline $\mathrm{GSH}^{\mathrm{a}}\left(\mu \mathrm{mol} \mathrm{I}^{-1}\right)$ & $2.6 \pm 0.6$ & $2.7 \pm 0.8$ & $2.7 \pm 0.9$ & $3.3 \pm 1.3$ & $P=0.003$ \\
\hline $\mathrm{G} \mathrm{PDH}^{\mathrm{a}}\left(\mathrm{Ug}^{-1} \mathrm{Hb}\right)$ & $8 \pm 1.9$ & $8.1 \pm 2$ & $8.2 \pm 2.1$ & $9.1 \pm 2$ & $P=0.04$ \\
\hline
\end{tabular}

Abbreviations: CAT, catalase; $\mathrm{CH}$, chronic hypertension; CPW, control pregnant women; GH, gestational hypertension; GPX, glutathione peroxidase; GRX, glutathione reductase; GSH, reduced glutathione;GSSG, oxidized glutathione; G6PDH, glucose-6-phosphate dehydrogenase; PCL, preeclampsia; SOD, superoxide-dismutase; TAS, total antioxidant status. glutathione
a $N=185$.

enzymatic activity levels were higher whereas G6PDH activity was lower in HDP groups than in PCW. Levels of nitrites/nitrates were increased in patients with preeclampsia as compared with the other groups. Finally, although malondialdehyde and ox-LDL levels were similar among groups, FFA were increased in all HDP as compared with PCW.

\section{DISCUSSION}

Our study shows an important imbalance between markers of oxidative stress and antioxidant defense systems in a large series of patients with different hypertensive disorders during pregnancy compared with PCW. This observation is shown by statistically significant lower levels of SOD, G6PDH, and GSH and higher levels of FFA, catalase, GPX, GRX and GSSG in hypertensive patients than in PCW. Nitrites/nitrate levels were elevated in preeclampsia compared with the other groups. In addition, although ox-LDL levels were not statistically different among the groups, it tended to be higher in all hypertensive groups than in PCW. Ox-LDL level is a marker of lipid peroxidation and its substrate, FFA, was elevated in all hypertensive groups. In contrast, we did not find any significant difference among groups in malondialdehyde levels.

The most important finding in our study was the alteration in the glutathione REDOX cycle not only in preeclampsia but also in all HDP. We found a significant reduction in GSH and at the same time an increase in GSSG, leading to a decrease in the GSH/GSSG ratio, and higher GPX and GRX activities in hypertensive groups compared with PCW. Diminished glutathione levels have been found previously in women with preeclampsia and GH. ${ }^{21,22}$ Regarding GPX, controversial results have been published; most studies suggest a total enzymatic activity lower in the placenta, whole blood, plasma and even in umbilical venous plasma, although any other studies have found higher levels in whole blood of preeclampsia or in plasma from patients with HELLP syndrome. ${ }^{3,6,23}$ The concentration of the specific GPX types (GPX 1-4) may have a role and explain in part these different results. ${ }^{3,6}$ In addition, it is not clear the cause for this disturbed GPX total activity and a decrease in protein translation or an increase in protein turnover have been proposed. ${ }^{3}$

Preeclampsia is partially attributed to lipid peroxidation of the syncytotrophoblast plasma membranes, and so markers of lipid peroxidation (such as malondialdehyde or isoprostanes) have been found to be increased in the plasma of women with preeclampsia in comparison with normal pregnancy. ${ }^{3,7}$ An efficient level of GSH is very important for the antioxidant protection of these membranes. GSH through its oxidation to GSSG by the enzyme GPX reduces $\mathrm{H}_{2} \mathrm{O}_{2}$ and lipid peroxides to water and lipidic alcohols, avoiding the oxidative damage. ${ }^{6,23,24}$ Although GSH can be partially recovered by recycling of GSSG by the action of GRX after an oxidative stress challenge, this mechanism appears to be of minor importance compared with GSH production via de novo synthesis. ${ }^{25}$ In the later process, a normal level of G6PDH is required; this is the key enzyme in the pentose phosphate pathway, as it provides the energy that is needed for GSH synthesis. ${ }^{22}$ Our results show a decrease in GSH levels with and increase in GSSG and in GPX and GRX activities in hypertensive groups; this may be the expression of an increased oxidative stress leading to depletion of GSH and the consequent increase in GSSG, that failed to recycle into GSH (even although GPX and GRX were increased as a compensatory mechanism). Probably, a part of the generated GSSG can be reduced back to GSH by the action of GRX, but the rate of GSH oxidation would exceed the rate of GSSG reduction. In addition, the lower level of G6PDH in our hypertensive patients supports a lack of synthesis of GSH as the most important factor in the GSH depletion. These data have never previously been described in HDP and they do not allow to establish if the impairment in the glutathione REDOX cycle is the cause or the consequence of an increased oxidative stress in these patients.

The noted underactivity of SOD has been previously reported in patients with preeclampsia, both in placentae and maternal 
blood. ${ }^{23,26}$ High amounts of $\mathrm{H}_{2} \mathrm{O}_{2}$ generated as a consequence of increased levels of superoxide anion, can promote an increment in catalase activity that would eliminate the peroxides. The previously described increment in lipolysis activity in preeclampsia ${ }^{27}$ is evident in our study with the significant increase noted in FFA levels in HDP as compared with PCW; promoted oxidative stress would allow LDL oxidation. A non-significant elevation of ox-LDL was found in HDP compared with PCW. A transient disturbance in $\gamma$-glutamyl-cysteinesynthetase activity (the enzyme that catalyzes the rate-limiting step of GSH synthesis) induced by the ox-LDL elevation, ${ }^{25}$ may also contribute, at least in part, to the diminished enzymatic activity to generate the novo GSH. Others authors have found high free cysteine concentration in pre-eclampsia and proposed that, because free cystein is the key amino acid in gluthathion synthesis, this may indicate an augmented gluthathion turnover to maintain physiological concentration of GSSG. ${ }^{4}$

Malondialdehyde, the most abundant reactive aldehyde derived of lipid peroxide breakdown, was one of the first biomarkers found elevated in preeclampsia. ${ }^{3,28}$ However, in this study, representing a large sample of HDP, we did not observe any significant difference in malondialdehyde levels between hypertensive and normotensive pregnant women; published papers have shown different results in this respect as no significant difference has been described previously ${ }^{29}$ but also other authors have found significant higher levels in preeclampsia compared with control group, with even higher levels when more severe disease. ${ }^{7}$ As FFA levels, other important marker of lipid oxidation, were increased in all hypertensive groups compared with PCW, it is probable that the lack of differences in malondialdehyde among the groups could be explained by the small sample in which this determination was available.

TAS reflects the redox status of the plasma, ${ }^{30}$ and it has been described to be decreased in preeclamptic patients compared with normotensive pregnants. ${ }^{3}$ Its lower level in $\mathrm{CH}$ as compared with the other studied groups is difficult to explain but may be in relationship with the chronic condition and longer evolution of $\mathrm{CH}$ as compared with $\mathrm{GH}$ or preeclampsia. In $\mathrm{CH}$ the disorders in the defense system may be the consequence of a failure in compensatory mechanisms. In preeclampsia, the lower fetal weight at birth can be the result of both fetal growth restriction and preterm delivery; this is well described in the literature. At the same time, fetal growth restriction may be also consequence of the increased oxidative stress in those women with preeclampsia; this has also been suggested in other studies, such as Fujimaki et al. ${ }^{31}$ Regarding nitric oxide levels in preeclampsia or in other hypertensive disorders, the results of different studies are contradictory; both elevated and diminished levels have been found. ${ }^{32,33}$ We found a significant increment in nitrate levels in preeclampsia and similar levels in the other hypertensive groups when compared with PCW.

Erythrocyte is one of the most commonly used experimental model for oxidation/antioxidation process study. SOD is in this context the main antioxidant-defending enzyme, due to its effect on the superoxide ion generated as a consequence of the hemoglobin autooxidation. GPX and GRX, which catalyze the interconversion of GSSG and GSH, have an important role together with catalase in the elimination of $\mathrm{H}_{2} \mathrm{O}_{2}$ and some other organic peroxides generated as a consequence of the physiological erythrocyte metabolism. ${ }^{34}$ The system with the described enzymes is one of the most relevant antioxidant physiological mechanisms and can be considered a reproducible marker of the oxidative stress status. ${ }^{35}$

Finally, although we cannot establish whether the changes we have observed in our study in antioxidant systems are the causes or the consequences of the increase in oxidative stress, we think that our results support the hypothesis that augmented oxidative stress occurs secondary to a failure of the defending capacity of the main antioxidant systems. We think that this imbalance may be responsible, at least in part, not only for the pathogenesis of preeclampsia but also for the other types of HDP. In preeclampsia, these disorders may be the result of the 'acute' development of endothelial dysfunction following disturbances in placentation and secondary systemic inflammatory response, whereas in $\mathrm{CH}$ they would more probably be the consequence of chronic endothelial dysfunction present previously to pregnancy. We also think that these alterations could be present even earlier than the disease is clinically evident, and in this way they could even be used as early biomarkers of future hypertensive complications of the pregnancy; however, more studies are needed to assess this subject.

\section{CONFLICT OF INTEREST}

The authors declare no conflict of interest.

\section{ACKNOWLEDGEMENTS}

This work was sponsored by a grant of the Consejería de Salud, Junta de Andalucía (Expte: 19/02).

1 Report of the National High Blood Pressure Education Program. Working Group on High Blood Pressure in Pregnancy. Am J Obstetr Gynecol 2000; 183: S1-S22.

2 Hauth JC, Cunninghan FJ. Preeclampsia- eclampsia. In Lindheimer MD, Roberts JM, Cunninghan FG (eds), Chesley's Hypertensive Disorders in Pregnancy, 2nd edn, Appleton and Lange: Stamford, CT, 1999, pp. 169-199.

3 Boutet M, Roland L, Thomas N, Bilodeau JF. Specific systemic antioxidant response to preeclampsia in late pregnancy: the study of intracellular glutathione peroxidases in maternal anf fetal blood. Am J Obstet Gynecol 2009; 200: 530.e1-530.e7.

4 Raijmakers MTM, Roes EM, Poston L, Steegers EAP, Peters WHM. The transient increase of oxidative stress during normal pregnancy is higher and persists afetr delivery in women with pre-eclampsia. Eur J Obstet Gynecol Reprod Biol 2008; 138: 39-44.

5 Roberts JM, Hubel CA. Is oxidative stress the link in the two-stage model of preeclampsia? Lancet 1999; 354: 788-789.

6 Mistry HD, Kurlak LO, Williams PJ, Ramsay MM, Symonds ME, Pipkin FB. Differential expression and distribution of placental glutathione peroxidases 1,3 and 4 in normal and preeclamptic pregnancy. Placenta 2010; 31: 401-408.

7 Sharma JB, Sharma A, Bahadur A, Vimala N, Satyam A, Mittal S. Oxidative stress markers and antioxidant levels in normal pregnancy and pre-eclampsia. Int J Gynaecol Obstet 2006; 94: 23-27.

8 Redman CWG, Sargent IL. Pre-eclampsia, the placenta and the maternal systemic inflammatory response - a review. Placenta 2003; 24: S21-S27.

9 Raijmakers MTM, Dechend R, Poston L. Oxidative stress and preeclampsia. Rationale for antioxidant clinical trials. Hypertension 2004; 44: 374-380.

10 Salonen JT, Yla-Herttuala S, Yamamoto R, Butler S, Korpela H, Salonen R, Nyyssonen K, Palinski W, Witztum JL. Autoantibody against oxidized LDL and progression of carotid atherosclerosis. Lancet 1992; 339: 883-887.

11 Mulder C, Schouten JA, Popp-Snijders C. Determination of free fatty acids: a comparative study of the enzymatic versus the gas chromatography and the colorimetric methods. J Clin Chem Clin Biochem 1983; 21: 823-827.

12 Green LC, Wagner DA, Glogowski J, Skipper PL, Wishnok JS, Tannenbaum SR. Analysis of nitrate, nitrite and (15N) nitrate in biological fluids. Anal Biochem 1982; 126: 131-138.

13 Miller NJ, Rice-Evans C, Davies MJ, Gopinathan V, Milner A. A novel method for measuring antioxidant capacity and its application to monitoring the antioxidant status in premature neonates. Clin Sci (Lon) 1993; 84: 407-412.

14 Paglia DE, Valentine WN. Studies on the quantitative and qualitative characterization of erythrocyte glutathione peroxidase. J Lab Clin Med 1967; 70: 158-169.

15 Goldberg DM, Spooner RJ. Methods of Enzymatic Analysis. Verlag Chemie: Dearfield Beach, FL, 1983, pp. 258-265.

16 McCord JM, Fridovich I. Superoxide dismutase. An enzymatic function for erythrocuprein (hemocuprein). J Biol Chem 1969; 244: 6049-6055.

17 Griffith OW. Determination of glutathione and glutathione disulfide using glutathione reductase and 2-vinylpyridine. Anal Biochem 1980; 106: 207-212.

18 Aebi HE. Methods of Enzymatic Analysis. Verlag Chemie: Dearfield Beach, FL, 1983, pp. 273-286.

19 Dahle LK, Hill EG, Holman RT. The thiobarbituric acid reaction and the autooxidation of polyunsaturated fatty acid methyl esters. Arch Biochem Biophys 1962; 98: 253-2261. 
20 Beutler E, Blume K, Kaplan JC, Lorh CW, Ramot B, Valentine WN. International Committee for standardization in haematology: recommended methods for red cells enzyme analysis. Br J Haematol 1997; 35: 331-340.

21 Kharb S. Low whole blood glutathione levels in pregnancies complicated by preeclampsia and diabetes. Clin Chem Acta 2000; 294: 179-183.

22 Nemeth I, Orvos H, Boda D. Blood glutathione redox status in gestational hypertension. Free Radic Biol Med 2001; 30: 715-721.

23 Perkins AV. Endogenous anti-oxidants in pregnancy and preeclapsia. Aust N Z J Obstet Gynaecol 2006; 46: 77-83.

24 Wassmann S, Wassmann K, Nickenig G. Modulation of oxidant and antioxidant enzyme expression and function in vascular cells. Hypertension 2004; 44: 381-386.

25 Shen L, Sevanian A. Ox- LDL induces macrophage gamma- GCS-HS protein expression: a role for ox-LDL associated lipid hydroperoxide in GSH synthesis. J Lipid Res 2001; 42: 813-823.

26 Aydin S, Benian A, Madazili R, Uludag S, Uzun H, Kaya S. Plasma malondialdehyde, superoxide dismutase, $\mathrm{SE}$-selectin, fibronectin, endothelin-1 and nitric oxide levels in women with preeclampsia. Eur J Obstetr Gynecol Reprod Biol 2004; 113. 21-25.

27 Hubel CA, Mclaughlin MK, Evans RW, Hauth BA, Sims CJ, Robert JM. Fasting serum triglycerides, free fatty acids, and malondialdehyde are increased in preeclampsia, are positively correlated, and decrease within 48 hours post partum. Am J Obstet Gynecol 1996; 174: 975-982.
28 Hubel CA, Roberts JM, Taylor RN, Musci TJ, Rogers GM, Mclaughlin MK. Lipid peroxidation in pregnancy: new perspectives on preeclampsia. Am J Obstetr Gynecol 1989; 161: 1025-1034.

29 Regan CL, Levine RJ, Baird DD, Ewell MG, Martz KL, Sibai BM, Rokach J, Lawson JA, Fitzgerald GA. No evidence for lipid peroxidation in severe preeclampsia. Am J Obstetr Gynecol 2001; 185: 572-578.

30 Erel 0 . A novel automated method to measure total antioxidant response against potent free radical reactions. Clin Biochem 2004; 37: 112-119.

31 Fujimaki A, Watanabe K, Mori T, Kimura C, Shinohara K, Wakatsuki A. Placental oxidative DNA damage and its repair in preeclamptic women with fetal growth restriction. Placenta 2011; 32: 367-372.

32 Ranta V, Viinikka L, Halmesmaki E, Ylikorkala O. Nitric oxide production with preeclampsia. Obstetr Gynecol 1999; 93: 442-445.

33 Seligman SP, Buyon JP, Clancy RM, Young BK, Abramson SB. The role of nitric oxide in the pathogenesis of preeclampsia. Am J Obstetr Gynecol 1994; 171: 944-948.

34 Ceballos-Picot I, Trivier JM, Nicole A, Sinet PN, Thevenin M. Age-correlated modifications of copper-zinc superoxide dismutase and glutathione-related enzyme activities in human erythrocytes. Clin Chem 1992; 38: 66-70.

35 Griffiths NR, Moller L, Bartosz G, Bast A, Bertoni-Freddari C, Collns A, Cooke M, Coolen S, Haenen G, Hoberg AM, Loft S, Lunec J, Olinski R, Parry J, Pompella A, Poulsen H, Verhagen H, Astley SB. Biomarkers. Mol Aspects Med 2002; 23: 101-208. 